\title{
On algebraic points in the plane near smooth curves
}

\author{
Vasili Bernik, Friedrich Götze, Olga Kukso
}

October 4, 2009

\section{Introduction}

Despite major results on the distribution of rational numbers on the real line there remain a number of deep problems. Some of them can be found in the monographs of Cassels and Schmidt $[1,2]$. The problem of counting integer points is a classical topic in number theory and there are various related problems like the Gauss circle problem or the problem number of divisors of natural numbers bounded by some big number [3, 4]. Some facts on counting integer points in multidimensional domains can be found in [5]. During the last 20 years considerable progress has been made concerning the number of points with rational coordinates near smooth curves by Beresnevich and Vilani $[6,7]$ insofar as the lower and upper bounds that have been obtained are of the same order.

In the present paper we introduce a method, which allows us to obtain bounds for the number of points with algebraic coordinates lying in a given domains of a Euclidean space. We consider algebraic points in the plane, but part of our results can be generalized to higher dimensional spaces.

Let $P \in \mathbb{Z}[x]$ be of the form

$$
\begin{gathered}
P(x)=P_{n}(x)=a_{n} x^{n}+a_{n-1} x^{n-1}+\cdots+a_{1} x+a_{0}, \\
H=H(P)=\max _{1 \leqslant j \leqslant n}\left|a_{j}\right|, \quad \operatorname{deg} P=n .
\end{gathered}
$$

Let $\mu A$ be the Lebesgue measure of a measurable set $A \subset \mathbb{R}^{2}$, and $|I|$ the length of an interval $I \subset \mathbb{R}$. In what follows $c, c(n), c_{1}, c_{2}, \ldots$ stand for some positive constants depending on $n$ only. Let $Q>Q_{0}(n)$, where $Q_{0}$ is a sufficiently large number. We will use the Vinogradov symbols $f \ll g$ which means that $f \leqslant c g$. The notation $B \asymp D$ means $D \ll B \ll D$. 
For some arbitrary positive constants $\mu_{1}, \mu_{2}$ consider a rectangle

$$
\Pi_{1}=I_{1} \times I_{2}=\left[a_{1}, b_{1}\right] \times\left[a_{2}, b_{2}\right] \subset\left[-\frac{1}{2}, \frac{1}{2}\right] \subset \mathbb{R}^{2}
$$

such that

$$
\Pi_{1} \cap\{|x-y| \leqslant 0.1\}=\emptyset
$$

and

$$
\left|I_{1}\right|=b_{1}-a_{1}=Q^{-\mu_{1}},\left|I_{2}\right|=b_{2}-a_{2}=Q^{-\mu_{2}} .
$$

Note that the lengths of $I_{1}$ and $I_{2}$ are small provided that $\mu_{1}>0, \mu_{2}>0$ and $Q$ is sufficiently large.

Suppose that $\alpha_{1}, \alpha_{2}, \ldots, \alpha_{k}$ denote $k$ real roots of $P, 1 \leqslant k \leqslant n$.

We introduce the class of polynomials

$$
\mathbf{P}_{n}(Q)=\left\{P_{n} \in \mathbb{Z}[x]: \operatorname{deg} P=n, n \geqslant 3, a_{n} \gg H(P), H(P) \leqslant Q\right\} .
$$

The condition $\left|a_{n}\right| \gg H$ implies that the roots of $P(x)$ are bounded, see Sprindzuk [8].

Let $K_{n}\left(\Pi_{1}, Q\right)$ be the set of points $\left(\alpha_{i}, \alpha_{j}\right), 1 \leqslant i<j \leqslant k$, such that

(i) $\left(\alpha_{i}, \alpha_{j}\right)$ are real roots of $P \in \mathbf{P}_{n}(Q)$,

(ii) $\left(\alpha_{i}, \alpha_{j}\right) \in \Pi_{1}$.

Remark. Condition (ii) excludes the coincidence of the roots $\alpha_{1}$ and $\alpha_{2}$.

The aim of this paper is to estimate the cardinality of $K_{n}\left(\Pi_{1}, Q\right)$.

Theorem 1 Let $0<\mu_{i}<\frac{1}{2}, i=1,2$. Then

$$
\# K_{n}\left(\Pi_{1}, Q\right) \gg Q^{n+1-\mu_{1}-\mu_{2}} .
$$

Remark. Consider $J_{1} \times J_{2}=\left[\frac{1}{3}-Q^{-1-\varepsilon}, \frac{1}{3}+Q^{-1-\varepsilon}\right] \times\left[\frac{1}{4}-Q^{-1-\varepsilon}, \frac{1}{4}+Q^{-1-\varepsilon}\right]$, where $\varepsilon>0$. Suppose that, on the contrary, that there is a polynomial $T \in \mathbf{P}_{n}(Q)$ such that a pair of its roots $\left(\alpha_{1}, \alpha_{2}\right)$ belongs to $J_{1} \times J_{2}$ and $T$ is coprime to $P(x)=(3 x-1)(4 x-1)=12 x^{2}-7 x+1$. The last assumption implies that $|R(T, P)| \geq 1$, where $R(T, P)$ is the resultant of $T(x)$ and $P(x)$. Since the roots of $T(x)$ are bounded, we have

$$
\begin{aligned}
& 1 \leqslant|R(T, P)|=12^{n} a_{n}^{2} \prod_{i=1}^{n}\left|\frac{1}{3}-\alpha_{i}\right| \prod_{j=1}^{n}\left|\frac{1}{4}-\alpha_{j}\right|= \\
&=12^{n} a_{n}^{2}\left|\alpha_{1}-\frac{1}{3}\right|\left|\alpha_{2}-\frac{1}{4}\right| \prod_{i \neq 1}\left|\frac{1}{3}-\alpha_{i}\right| \prod_{j \neq 2}\left|\frac{1}{4}-\alpha_{j}\right| \ll \\
& \ll Q^{2} Q^{-1-\varepsilon} Q^{-1-\varepsilon}=Q^{-2 \varepsilon} .
\end{aligned}
$$


The inequality (5) yields a contradiction if $Q$ is sufficiently large.

This remark shows that Theorem 1 cannot be considerably improved. It won't hold for $\max _{j} \mu_{j}>1$. Improvements are possible for intervals $I_{1}, I_{2}$ only that don't contain algebraic numbers of small degree and height.

Corollary. Let $f(x)$ be a continuous function on the interval $I=[a, b]$ and let

$$
\mathcal{L}(Q, \lambda)=\left\{(x, y): x \in I,|y-f(x)|<Q^{-\lambda}\right\}, \quad 0<\lambda<\frac{1}{2} .
$$

Then there are at least $c(n) Q^{n+1-\lambda}$ algebraic points such that $\left(\alpha_{1}, \alpha_{2}\right) \in$ $\mathcal{L}(Q, \lambda)$.

Proof of the corollary. The set $\mathcal{L}(Q, \lambda)$ represents a strip containing the curve $y=f(x)$. Its width equals $2 Q^{-\lambda}, 0<\lambda<\frac{1}{2}$. Let us split an interval $[a, b]$ into equal parts of length at most $Q^{-\lambda}$ choosing points

$$
x_{0}=a, x_{1}=x_{0}+Q^{-\lambda}, \ldots, x_{j}=x_{j-1}+Q^{\lambda}, \ldots, x_{s}=x_{0}+s Q^{\lambda},
$$

where $\lambda \leqslant 1$. Furthermore, inscribe rectangles of size $Q^{-\lambda} \times c(n) Q^{-\lambda}$ into every rectangle

$$
\left\{(x, y):\left|x-\frac{x_{i}+x_{i+1}}{2}\right| \leqslant \frac{1}{2} Q^{-\lambda},|y-f(x)|<\frac{1}{2} Q^{-\lambda}\right\} .
$$

By Theorem 1, every such rectangle contains at least $c(n) Q^{n+1-2 \lambda}$ algebraic points $\left(\alpha_{1}, \alpha_{2}\right)$. Collecting the algebraic in all rectangles we obtain

$$
\# \mathcal{L}(Q, \lambda) \cap \mathbb{A}_{n} \gg c(n) Q^{n+1-\lambda} .
$$

The proof of Theorem 1 is based on the construction of special polynomials $P(t) \in \mathbf{P}_{n}(Q)$ such that

1. $|P(x)|$ and $|P(y)|$ are small,

2. $\left|P^{\prime}(x)\right|$ and $\left|P^{\prime}(y)\right|$ are comparable with $H(P)$,

where $(x, y) \in B_{1} \subset \Pi_{1}$ and $\mu B_{1}>\frac{1}{2} \mu \Pi_{1}$.

Let $\bar{c}=\left(c_{1}, c_{2}, c_{3}, c_{4}\right)$ and $\bar{v}=\left(v_{1}, v_{2}\right)$ denote positive vectors. Let $M_{n}(\bar{c}, Q)$ denote the set of points $\bar{x} \in \Pi_{1}$ such that the following system

$$
\left\{\begin{array}{l}
|P(x)|<c_{1} Q^{-v_{1}} \\
|P(y)|<c_{2} Q^{-v_{2}} \\
\left|P^{\prime}(x)\right|<c_{3} Q \\
\left|P^{\prime}(y)\right|<c_{4} Q \\
v_{1}+v_{2}=n-1
\end{array}\right.
$$

has a solution $P(t) \in \mathbb{Z}[t] \backslash\{0\}$. 
Theorem 2 Assume that $c_{1} c_{2} \min \left(c_{3}, c_{4}\right)<2^{-n-38} n^{-2}$ and $\max \left(c_{1}, c_{2}, c_{3}, c_{4}\right) \leq 1$. Then

$$
\mu M_{n}(\bar{c}, Q)<\frac{1}{4}\left|I_{1}\right|\left|I_{2}\right|
$$

To prove Theorem 2 we impose an extra condition on $P$. We consider only irreducible polynomials. This condition is not very restrictive and leads to an equivalent problem as shown in Sprindzuk and Bernik [8, 9].

\section{Auxiliary statements}

This section contains several lemmas that will be used in the proof of Theorem 2 .

In what follows $\mathcal{P}_{n}(Q)$ denotes the class of irreducible polynomials $P(t)$ with $H(P) \leqslant Q$ such that $(7)$ holds. Furthermore, let $\tilde{\mathcal{P}}_{n}(H)$ be the subclass of $\mathcal{P}_{n}(H)$ consisting of polynomials $P$ with $H(P)=H$.

For each polynomial $P \in \tilde{\mathcal{P}}_{n}(H)$ with roots $\alpha_{1}, \alpha_{2}, \ldots, \alpha_{n}$, we pick a pair of roots $\alpha_{i}$ and $\alpha_{j}, i \neq j$. Throughout for convenience, we shall write $\alpha_{1}$ instead of $\alpha_{i}$ and $\beta_{1}$ instead of $\alpha_{j}$. Furthermore, we order the other roots of $P$ with respect to the distance from the roots $\alpha_{1}$ and $\beta_{1}$

$$
\begin{gathered}
\left|\alpha_{1}-\alpha_{2}\right| \leqslant\left|\alpha_{1}-\alpha_{3}\right| \leqslant \cdots \leqslant\left|\alpha_{1}-\alpha_{n}\right|, \\
\left|\beta_{1}-\beta_{2}\right| \leqslant\left|\beta_{1}-\beta_{3}\right| \leqslant \cdots \leqslant\left|\beta_{1}-\beta_{n}\right| .
\end{gathered}
$$

Obviously, in (9), the set $\beta_{1}, \beta_{2}, \ldots, \beta_{n}$ is a permutation of the roots $\alpha_{1}, \alpha_{2}, \ldots, \alpha_{n}$. Denote

$$
\begin{aligned}
& S\left(\alpha_{1}\right)=\left\{x \in \mathbb{R}:\left|x-\alpha_{1}\right|=\min _{1 \leqslant j \leqslant n}\left|x-\alpha_{j}\right|\right\}, \\
& S\left(\beta_{1}\right)=\left\{x \in \mathbb{R}:\left|x-\beta_{1}\right|=\min _{1 \leqslant j \leqslant n}\left|x-\beta_{j}\right|\right\} .
\end{aligned}
$$

We will consider now the system of inequalities (7) for $x \in S\left(\alpha_{1}\right)$ and $y \in$ $S\left(\beta_{1}\right)$.

Lemma 1 (see [8]) If $\left|a_{n}\right| \gg H$ then for any $i, 1 \leqslant i \leqslant n$,

$$
\left|\alpha_{i}\right|<c
$$

Lemma 2 Let $P \in \tilde{\mathcal{P}}_{n}(H)$ and $x \in S\left(\alpha_{1}\right)$. Then

$$
\left|x-\alpha_{1}\right| \leqslant n \frac{|P(x)|}{\left|P^{\prime}(x)\right|},
$$




$$
\begin{gathered}
\left|x-\alpha_{1}\right| \leqslant 2^{n-1}|P(x)|\left|P^{\prime}\left(\alpha_{1}\right)\right|^{-1} \\
\left|x-\alpha_{1}\right| \leqslant \min _{2 \leqslant j \leqslant n}\left(2^{n-j}|P(x)|\left|P^{\prime}\left(\alpha_{1}\right)\right|^{-1} \prod_{k=2}^{j}\left|\alpha_{1}-\alpha_{k}\right|\right)^{\frac{1}{j}} .
\end{gathered}
$$

The first inequality in (10) immediately follows from the identity $\left|P^{\prime}(x)\right||P(x)|^{-1}=\left|\sum_{i=1}^{n} \frac{1}{\left(x-\alpha_{i}\right)}\right|$ and the inequalities $\left|x-\alpha_{1}\right| \leqslant\left|x-\alpha_{j}\right|$, $j=2, \ldots, n$. The remaining inequalities were proved in Sprindzuk and Bernik $[8,10]$.

Let $\varepsilon>0$ be sufficiently small, and let $N=N(n)>0$ be sufficiently large fixed numbers. Write $\varepsilon_{1}=\varepsilon N^{-1}$, and $T=\left[\varepsilon_{1}\right]^{-1}$.

Using (9) define numbers $\rho_{1, j}$ and $\rho_{2, j}(2 \leqslant j \leqslant n)$ by setting

$$
\begin{aligned}
& \left|\alpha_{1}-\alpha_{j}\right|=H^{-\rho_{1 j}}, \rho_{1, n} \leqslant \cdots \leqslant \rho_{12}, \\
& \left|\beta_{1}-\beta_{j}\right|=H^{-\rho_{2 j}}, \rho_{2, n} \leqslant \cdots \leqslant \rho_{22} .
\end{aligned}
$$

By Lemma 1 the roots $\alpha_{j}$ are bounded. Then the inequalities (9) and (11) imply $\rho_{i, j}>-\frac{\varepsilon_{1}}{2}$.

For every polynomial there are uniquely determined integral vectors $\left(k_{2}, k_{3}, \ldots, k_{n}\right)$ and $\left(l_{2}, l_{3}, \ldots, l_{n}\right)$ such that the inequalities

$$
\begin{gathered}
\left(k_{j}-1\right) T^{-1} \leqslant \rho_{1 j}<k_{j} T^{-1}, \quad 0 \leqslant k_{n} \leqslant \ldots \leqslant k_{2}, \\
\left(l_{j}-1\right) T^{-1} \leqslant \rho_{2 j}<l_{j} T^{-1}, \quad 0 \leqslant l_{n} \leqslant \ldots \leqslant l_{2}
\end{gathered}
$$

hold. Furthermore, define

$$
q_{i}=T^{-1} \sum_{m=i+1}^{n} k_{m}, \quad r_{i}=T^{-1} \sum_{m=i+1}^{n} l_{m}, 1 \leq i \leq n-1 .
$$

Consider $\cup_{H=1}^{\infty} \tilde{\mathcal{P}}_{n}(H)$. Using results of Sprindzuk [8], the number of possible vectors $\bar{k}=\left(k_{2}, k_{3}, \ldots, k_{n}\right)$ and $\bar{l}=\left(l_{2}, l_{3}, \ldots, l_{n}\right)$ is finite.

Thus, all polynomials $P \in \tilde{\mathcal{P}}_{n}(H)$ corresponding to the same pair of vectors $\bar{s}=(\bar{k}, \bar{l})$ can be grouped together into a class $\tilde{\mathcal{P}}_{n}(H, \bar{s})$.

Lemma 3 (see Bernik [10]) Let $P \in \tilde{\mathcal{P}}_{n}(H, \bar{s})$. The we have

$$
\begin{aligned}
& H^{1-q_{1}} \leqslant\left|P^{\prime}\left(\alpha_{1}\right)\right|<H^{1-q_{1}+(n-1) \varepsilon_{1}}, \\
& H^{1-r_{1}} \leqslant\left|P^{\prime}\left(\beta_{1}\right)\right|<H^{1-r_{1}+(n-1) \varepsilon_{1}},
\end{aligned}
$$

and for any $k, 2 \leqslant k \leqslant n$,

$$
\begin{aligned}
& \left|P^{(k)}\left(\alpha_{1}\right)\right| \ll H^{1-q_{k}+k(n-1) \varepsilon_{1}} \\
& \left|P^{(k)}\left(\beta_{1}\right)\right| \ll H^{1-r_{k}+k(n-1) \varepsilon_{1}} .
\end{aligned}
$$


Lemma 4 Let $\delta, K_{0}, \eta_{1}, \eta_{2} \in \mathbb{R}_{+}$. Furthermore, let $P_{1}, P_{2} \in \mathbb{Z}[x]$ be two relatively prime polynomials of degree at most $n$ with $\max \left(H\left(P_{1}\right), H\left(P_{2}\right)\right) \leqslant$ $K$ and $K>K_{0}(\delta)$. Let $J_{1}$ and $J_{2}$ denote intervals with $\left|J_{1}\right|=K^{-\eta_{1}},\left|J_{2}\right|=$ $K^{-\eta_{2}}$. If there exist numbers $\tau_{1}, \tau_{2}>0$ such that for all $(x, y) \in J_{1} \times J_{2}$

$$
\begin{gathered}
\max \left(\left|P_{1}(x)\right|,\left|P_{2}(x)\right|\right)<K^{-\tau_{1}}, \\
\max \left(\left|P_{1}(y)\right|,\left|P_{2}(y)\right|\right)<K^{-\tau_{2}},
\end{gathered}
$$

then

$$
\tau_{1}+\tau_{2}+2+2 \max \left(\tau_{1}+1-\eta_{1}, 0\right)+2 \max \left(\tau_{2}+1-\eta_{2}, 0\right)<2 n+\delta .
$$

For the proof see Bernik [11].

Remark. Actually, a stronger result holds, namely

$$
\tau_{1}+\tau_{2}+2+2 \max \left(\sum_{k=1}^{\infty} \tau_{1}+1-\eta_{1}, 0\right)+2 \max \left(\sum_{k=1}^{\infty} \tau_{2}+1-\eta_{2}, 0\right)<2 n+\delta .
$$

When we apply Lemma 4 we will usually choose parameters $\tau_{1}, \tau_{2}, \eta_{1}, \eta_{2}$ satisfying

$$
\tau_{1}=k_{2} T^{-1}+q_{1}-1, \tau_{2}=l_{2} T^{-1}+r_{1}-1, \eta_{1}=k_{2} T^{-1}, \eta_{2}=l_{2} T^{-1} .
$$

Thus, if the difference between, say, $l_{2} T^{1}$ and $r_{1}$ is larger, then the result of Lemma 4 will be stronger. Therefore, without loss of generality, we can assume that $k_{2} T^{-1}=q_{1}, l_{2} T^{-1}=r_{1}$, and $q_{j}=r_{j}=0$ for $j \geq 2$.

\section{Proof of Theorem 2}

First, we consider a special case of system (7) when $\left|P^{\prime}(x)\right|,\left|P^{\prime}(y)\right|$ are bounded below. Let us remind that $x \in S\left(\alpha_{1}\right)$ and $y \in S\left(\beta_{1}\right)$.

Proposition 1. Let $v>\frac{1}{2}$ denote a constant and let $M_{n, 1}(\bar{c}, Q)$ denote the set of solutions $(x, y) \in I_{1} \times I_{2}$ of the system

$$
\left\{\begin{array}{l}
|P(x)| \leq c_{1} Q^{-v_{1}} \\
|P(y)| \leq c_{2} Q^{-v_{2}} \\
Q^{v}<\left|P^{\prime}(x)\right|<c_{3} Q \\
Q^{v}<\left|P^{\prime}(y)\right|<c_{4} Q .
\end{array}\right.
$$

Then

$$
\mu M_{n, 1}(\bar{c}, \bar{v}, Q)<\frac{1}{8}\left|I_{1}\right|\left|I_{2}\right|
$$


Now estimates for $\left|P^{\prime}(x)\right|$ and $\left|P^{\prime}(y)\right|$ provide estimates for $\left|P^{\prime}\left(\alpha_{1}\right)\right|$ and $\left|P^{\prime}\left(\beta_{1}\right)\right|$.

By the first inequality in (10) for any $x \in S\left(\alpha_{1}\right)$ and $y \in S\left(\beta_{1}\right)$, we have

$$
\begin{aligned}
& \left|x-\alpha_{1}\right|<n|P(x)|\left|P^{\prime}(x)\right|^{-1}<c_{1} n Q^{-v_{1}-v}, \\
& \left|y-\beta_{1}\right|<n|P(y)|\left|P^{\prime}(y)\right|^{-1}<c_{2} n Q^{-v_{2}-v} .
\end{aligned}
$$

The Mean Value Theorem yields

$$
\begin{array}{cc}
P^{\prime}(x)=P^{\prime}\left(\alpha_{1}\right)+P^{\prime \prime}\left(\xi_{1}\right)\left(x-\alpha_{1}\right) & \text { for some } \xi_{1} \in\left(\alpha_{1}, x\right), \\
P^{\prime}(y)=P^{\prime}\left(\beta_{1}\right)+P^{\prime \prime}\left(\xi_{2}\right)\left(y-\beta_{2}\right) & \text { for some } \xi_{2} \in\left(\beta_{2}, y\right) .
\end{array}
$$

Obviously, we have $\left|P^{\prime \prime}\left(\xi_{1}\right)\left(x-\alpha_{1}\right)\right| \ll Q^{1-v_{1}-v},\left|P^{\prime \prime}\left(\xi_{2}\right)\left(y-\beta_{1}\right)\right| \ll$ $Q^{1-v_{2}-v}$. Thus, for sufficiently large $Q$ we obtain

$$
\begin{aligned}
& \frac{3}{4} Q^{v} \leq \frac{3}{4}\left|P^{\prime}(x)\right|<\left|P^{\prime}\left(\alpha_{1}\right)\right|<\frac{4}{3}\left|P^{\prime}(x)\right| \leq \frac{4}{3} c_{3} Q, \\
& \frac{3}{4} Q^{v} \leq \frac{3}{4}\left|P^{\prime}(y)\right|<\left|P^{\prime}\left(\beta_{1}\right)\right|<\frac{4}{3}\left|P^{\prime}(y)\right| \leq \frac{4}{3} c_{4} Q .
\end{aligned}
$$

By (14) and Lemma 2, we have

$$
\begin{aligned}
& \left|x-\alpha_{1}\right|<\frac{4}{3} n|P(x)|\left|P^{\prime}\left(\alpha_{1}\right)\right|^{-1}, \\
& \left|y-\beta_{1}\right|<\frac{4}{3} n|P(y)|\left|P^{\prime}\left(\beta_{1}\right)\right|^{-1} .
\end{aligned}
$$

Let $\sigma_{x}(P), \sigma_{y}(P)$ denote the sets of solutions of (15) for $x$ and $y$, respectively. Let $\Pi_{2}(P)=\sigma_{x}(P) \times \sigma_{y}(P)$. Clearly, all solutions $(x, y) \in$ $S\left(\alpha_{1}\right) \times S\left(\beta_{1}\right)$ of the system $(12)$ are contained in $\Pi_{2}(P)$.

We introduce the intervals

$$
\begin{aligned}
\sigma_{1 x}(P): & \left|x-\alpha_{1}\right|<c_{5} Q^{-\gamma}\left|P^{\prime}\left(\alpha_{1}\right)\right|^{-1} \\
\sigma_{1 y}(P): & \left|y-\beta_{1}\right|<c_{5} Q^{-\gamma}\left|P^{\prime}\left(\beta_{1}\right)\right|^{-1}
\end{aligned}
$$

where values of positive constants $\gamma$ and $c_{5}$ will be specified below. Assign $\Pi_{3}(P)=\sigma_{1 x}(P) \times \sigma_{1 y}(P)$.

Now we shall estimate the values of $P$ and $P^{\prime}$ on the intervals $\sigma_{1 x}(P)$ and $\sigma_{1 y}(P)$. For the sake of simplicity we shall consider $P(y)$ and $P^{\prime}(y)$ on $\sigma_{1 y}(P)$ only. The Mean Value Theorem yields

$$
\begin{array}{lccc}
P(y)= & P^{\prime}\left(\beta_{1}\right)\left(y-\beta_{1}\right)+\frac{1}{2} P^{\prime \prime}\left(\xi_{3}\right)\left(y-\beta_{1}\right)^{2} & \text { for some } & \xi_{3} \in\left(\beta_{1}, y\right), \\
P^{\prime}(y)= & P^{\prime}\left(\beta_{1}\right)+P^{\prime \prime}\left(\xi_{4}\right)\left(y-\beta_{1}\right) & \text { for some } & \xi_{4} \in\left(\beta_{1}, y\right) .
\end{array}
$$

By (14) and (16), the second terms of $P(y)$ and $P^{\prime}(y)$ may be estimated as follows

$$
\begin{aligned}
\left|\frac{1}{2} P^{\prime \prime}\left(\xi_{3}\right)\left(y-\beta_{1}\right)^{2}\right| & \ll Q^{1-2 \gamma-2 v}, \\
\left|P^{\prime \prime}\left(\xi_{4}\right)\left(y-\beta_{1}\right)\right| & \ll Q^{1-\gamma-v} .
\end{aligned}
$$


From (17) and (18) we get

$$
\begin{gathered}
|P(y)|<\frac{4}{3} c_{5} Q^{-\gamma} \\
\left|P^{\prime}(y)\right|<\frac{5}{3} c_{4} Q .
\end{gathered}
$$

Similarly, for $P(x)$ and $P^{\prime}(x)$ on interval $\sigma_{1 x}(P)$ we obtain

$$
\begin{gathered}
|P(x)|<\frac{4}{3} c_{5} Q^{-\gamma}, \\
\left|P^{\prime}(x)\right|<\frac{5}{3} c_{3} Q .
\end{gathered}
$$

Fix the vector $\bar{b}=\left(a_{n}, \ldots, a_{3}\right)$ of coefficients of $P(x)$. The polynomials $P \in \tilde{\mathcal{P}}_{n}(H, \bar{s})$ with the same vector $\bar{b}$ form a subclass $\mathcal{P}(\bar{b})$.

Without loss of generality, we may assume that $a_{n}>0$. Otherwise multiply the polynomial by -1 which does not change the system (7). Every coefficient $a_{j},(3 \leqslant j \leqslant n-1)$ may take at most $(2 \mathrm{Q}+1)$ values. Thus we have $\# \mathcal{P}(\bar{b}) \leq Q(2 Q+1)^{n-3}$. For convenience, note that $\# \mathcal{P}(\bar{b}) \leq 2^{n-1} Q^{n-2}$.

We consider two types of rectangles $\Pi_{3}(P)$. One type of rectangle $\Pi_{3}\left(P_{1}\right)$ with $P_{1} \in \mathcal{P}(\bar{b})$ is called inessential if there is another rectangle $\Pi_{3}\left(P_{2}\right)$ with $P_{2} \in \mathcal{P}(\bar{b})$ such that

$$
\mu\left(\Pi_{3}\left(P_{1}\right) \cap \Pi_{3}\left(P_{2}\right)\right) \geqslant 0.5 \mu\left(\Pi_{3}\left(P_{1}\right)\right) .
$$

The other type of rectangle $\Pi_{3}\left(P_{1}\right)$ and is called essential. It satisfies: for any $P_{2} \in \mathcal{P}(\bar{b})$ different from $P_{1}$

$$
\mu\left(\Pi_{3}\left(P_{1}\right) \cap \Pi_{3}\left(P_{2}\right)\right)<0.5 \mu\left(\Pi_{3}\left(P_{1}\right)\right) .
$$

The case of essential rectangles. Summing the measures of rectangles for all polynomials in $\mathcal{P}(\bar{b})$, we obtain

$$
\sum_{P \in \mathcal{P}(\bar{b})} \mu \Pi_{3}(P) \leqslant 2\left|I_{1}\right| \times\left|I_{2}\right|
$$

Combining the definitions of $\sigma_{1 x}(P), \sigma_{1 y}(P), \sigma_{x}(P), \sigma_{y}(P)$ (see $(15),(16)$ ), we get

$$
\begin{aligned}
& \mu \sigma_{x}(P)<\frac{4}{3} n c_{1} c_{5}^{-1} Q^{-v_{1}+\gamma} \mu \sigma_{1 x}(P), \\
& \mu \sigma_{y}(P)<\frac{4}{3} n c_{2} c_{5}^{-1} Q^{-v_{2}+\gamma} \mu \sigma_{1 y}(P) .
\end{aligned}
$$

Let us estimate the measure of the union of $\Pi_{2}(P)$ for all polynomials 
selected above.

$$
\begin{gathered}
\sum_{P \in \mathcal{P}(\bar{b})} \mu \Pi_{2}(P)=\sum_{P \in \mathcal{P}(\bar{b})} \mu \sigma_{x}(P) \times \mu \sigma_{y}(P)< \\
<\sum_{P \in \mathcal{P}(\bar{b})} 2 n^{2} c_{1} c_{2} c_{5}^{-2} Q^{-v_{1}-v_{2}+2 \gamma} \mu \sigma_{1 x}(P) \times \mu \sigma_{1 y}(P)= \\
=2 n^{2} c_{1} c_{2} c_{5}^{-2} Q^{-v_{1}-v_{2}+2 \gamma} \sum_{P \in \mathcal{P}(\bar{b})} \mu \Pi_{3}(P)< \\
<4 n^{2} c_{1} c_{2} c_{5}^{-2} Q^{-v_{1}-v_{2}+2 \gamma}\left|I_{1}\right|\left|I_{2}\right| .
\end{gathered}
$$

Summing over $\bar{b}$, we get

$$
\sum_{\bar{b}} \sum_{P \in \mathcal{P}(\bar{b})} \mu \Pi_{2}(P)<2^{n+1} n^{2} c_{1} c_{2} c_{5}^{-2} Q^{n-2-v_{1}-v_{2}+2 \gamma}\left|I_{1}\right|\left|I_{2}\right| .
$$

Taking into account $v_{1}+v_{2}=n-1$, and writing $\gamma=\frac{1}{2}$, we obtain

$$
\sum_{\bar{b}} \sum_{P \in \mathcal{P}(\bar{b})} \mu \Pi_{2}(P)<2^{n+1} n^{2} c_{1} c_{2} c_{5}^{-2}\left|I_{1}\right|\left|I_{2}\right|
$$

Given $c_{5}^{2}=2^{n+5} n^{2} c_{1} c_{2}$, the estimate in (25) does not exceed $2^{-4}\left|I_{1}\right|\left|I_{2}\right|$.

\section{The case of inessential rectangles.}

Define $R(t)=P_{2}(t)-P_{1}(t)=b_{2} t^{2}+b_{1} t+b_{0}$. Without loss of generality, assume $b_{2} \geq 0$. Obviously, $R(t)$ is not identically zero. The Conditions (19), $(20)$, and $P_{1}, P_{2} \in \mathcal{P}(\bar{b})$ imply

$$
\begin{aligned}
|R(x)| & =\left|b_{2} x^{2}+b_{1} x+b_{0}\right|<3 c_{5} Q^{-\gamma}, \\
\left|R^{\prime}(x)\right| & =\left|2 b_{2} x+b_{1}\right|<3 c_{3} Q, \\
|R(y)| & =\left|b_{2} y^{2}+b_{1} y+b_{0}\right|<3 c_{5} Q^{-\gamma} \\
\left|R^{\prime}(y)\right| & =\left|2 b_{2} y+b_{1}\right|<3 c_{4} Q .
\end{aligned}
$$

Let $\alpha$ and $\beta$ denote roots of the polynomial $R(x)$ with $\operatorname{deg} R=2$. By inequalities (26) for $|R(x)|,|R(y)|$, and Lemma 2, we can estimate

$$
\begin{aligned}
& |x-\alpha|<6 c_{5} Q^{-\gamma}\left|R^{\prime}(\alpha)\right|^{-1} \\
& |y-\beta|<6 c_{5} Q^{-\gamma}\left|R^{\prime}(\beta)\right|^{-1} .
\end{aligned}
$$

By (2), if $|\alpha-\beta|<0.08$, we arrive at a contradiction for sufficiently large $Q$

$$
0,1<|x-y| \leq|x-\alpha|+|y-\beta|+|\alpha-\beta|<0,09 .
$$


Thus $|\alpha-\beta| \geq 0.08$ and

$$
\left|R^{\prime}(\alpha)\right|=\left|R^{\prime}(\beta)\right|=b_{2}|\alpha-\beta|>0.08 b_{2} .
$$

Suppose $c_{4}=\min \left(c_{3}, c_{4}\right)$. Applying the Mean Value Theorem on the interval $\sigma_{1 y}$, we obtain

$$
R^{\prime}(y)=R^{\prime}(\beta)+R^{\prime \prime}\left(\xi_{5}\right)(y-\beta) \quad \text { for some } \xi_{5} \in[\beta, y] .
$$

Since $\left|R^{\prime \prime}\left(\xi_{5}\right)(y-\beta)\right|<24 c_{5} Q^{1-\gamma}\left|R^{\prime}(\beta)\right|^{-1}$, if $\left|R^{\prime}(\beta)\right|^{2}>48 c_{5} Q^{1-\gamma}$, then

$$
\left|R^{\prime}(\beta)\right|<2\left|R^{\prime}(y)\right|<6 c_{4} Q \text {. }
$$

The estimate (30) follows from the inequalities (14). This implies that the number of possible $b_{2}$ is bounded by

$$
\# b_{2}<75 c_{4} Q \text {. }
$$

Suppose that $I_{1}=\left[d_{1}, d_{2}\right], I_{2}=\left[f_{1}, f_{2}\right]$, and $\left|I_{2}\right| \geq\left|I_{1}\right|$.

First let us assume that $\left|I_{1}\right|=\left|I_{2}\right|=Q^{-\mu_{1}}$. The point $-\frac{b_{1}}{2 b_{2}}$ is the maximum of the parabola $z=b_{2} x^{2}+b_{1} x+b_{0}$. It is easy to verify that this point lies inside the interval $\left[\frac{d_{1}+d_{2}}{2}, \frac{f_{1}+f_{2}}{2}\right]$. The conditions $x \in I_{1} \subset\left[-\frac{1}{2}, \frac{1}{2}\right]$, $y \in I_{2} \subset\left[-\frac{1}{2}, \frac{1}{2}\right]$ imply

$$
\# b_{1} \leq 2 b_{2} Q^{-\mu_{1}}+2=2 b_{2}\left|I_{1}\right|+2
$$

and $\left|b_{1}\right| \leq\left|b_{2}\right|$.

Now assume $\left|I_{1}\right|>\left|I_{2}\right|$. Divide $I_{2}$ into $m=\left[\frac{\left|I_{2}\right|}{\left|I_{1}\right|}\right]+1$ intervals $J_{i}$ such that $J_{i} \leq\left|I_{1}\right|$ where $1 \leqslant j \leqslant m$. Similarly, for every pair $x \in I_{1}$ and $y \in J_{i}$ we obtain an upper bound for $\# b_{1}$ similar to (32). Summing (32) over $j$ gives the following exact estimate of the number of possible $b_{1}$

$$
\# b_{1} \leq\left(2 b_{2}\left|I_{1}\right|+2\right)\left(\left|I_{2}\right|\left|I_{1}\right|^{-1}+1\right) \leq 4 b_{2}\left|I_{2}\right| .
$$

Suppose now that (26) holds for some $R_{1}=b_{2} x^{2}+b_{1} x+b_{0}$. If we take $R_{2}=b_{2} x^{2}+b_{1} x+b_{0}+1$ we may shift the argument by $\Delta x$, i.e.,

$1=R_{2}(x)-R_{1}(x)=R_{1}(x+\Delta x)-R_{1}(x)=R^{\prime}\left(\xi_{6}\right) \Delta x$ for some $\xi_{6} \in[x, x+\Delta x]$.

If $x+\Delta x \in I_{1}$, then $\xi \in I_{1}$. For a fixed pair $\left(b_{2}, b_{1}\right)$ the estimate for the derivative in (26) can be improved, namely

$$
\left|R^{\prime}\left(\xi_{6}\right)\right|=\left|2 b_{2} \xi_{6}+b_{1}\right| \leq 2\left|b_{2}\right| \frac{1}{2}+\left|b_{1}\right| \leq 2\left|b_{2}\right| .
$$


Summarizing, we conclude that

$$
\Delta=\left|R^{\prime}\left(\xi_{6}\right)\right|^{-1} \geq \frac{1}{2}\left|b_{2}\right|^{-1} .
$$

This means that the number of possible values of $b_{0}$ is at most

$$
\# b_{0} \leqslant\left|I_{1}\right||\Delta|^{-1}<2\left|b_{2}\right|\left|I_{1}\right| .
$$

By Lemma 2 and the estimates $\left|R^{\prime}(\alpha)\right|>2^{-4} b_{2},\left|R^{\prime}(\beta)\right|>2^{-4} b_{2}$ from (26), we obtain

$$
|x-\alpha|<2^{8} c_{5} Q^{-\gamma} b_{2}^{-1}
$$

and

$$
|y-\beta|<2^{8} c_{5} Q^{-\gamma} b_{2}^{-1} .
$$

Thus, the measure of the intersection $\Pi_{3}\left(P_{1}\right) \cap \Pi_{3}\left(P_{2}\right)$ is less than $2^{18} c_{5}^{2} b_{2}^{-2} Q^{-2 \gamma}$. If $\gamma=\frac{1}{2}$, then the measure of the inessential rectangle is less than

$$
2^{19} c_{5}^{2} b_{2}^{-2} Q^{-1}
$$

Using the estimates for $b_{0}, b_{1}, b_{2}$ from $(31),(33),(34)$, we may sum (35) over $\left(b_{0}, b_{1}, b_{2}\right)$, and get

$$
\sum_{b_{2}} \sum_{b_{1}} \sum_{b_{0}} \mu \Pi_{3}(P)<2^{29} \min \left(c_{3}, c_{4}\right) c_{5}^{2}\left|I_{1}\right|\left|I_{2}\right| .
$$

For $c_{5}=2^{n+5} n^{2} c_{1} c_{2}$ the estimate in (36) says

$$
2^{n+34} n^{2} c_{1} c_{2} \min \left(c_{3}, c_{4}\right)\left|I_{1}\right|\left|I_{2}\right| .
$$

Given $c_{1} c_{2} \min \left(c_{3}, c_{4}\right)<2^{-n-38} n^{-2}$, this bound is smaller than $2^{-4}$. Thus, we proved that

$$
\mu M_{n 1}(\bar{c}, Q)<\frac{1}{8}\left|I_{1}\right|\left|I_{2}\right| .
$$

The remaining part of the proof strongly depends on the structures of $\bar{q}$, $\bar{r}$ (they were introduced in the Auxiliary Statements) and on their relations with the degrees $v_{1}, v_{2}$. In all of these statements below the measure tends to zero as $Q \rightarrow \infty$. The constants $c_{1}, c_{2}, c_{3}, c_{4}$, and others no longer play a significant role and will be replaced by the Vinogradov symbol $\ll$ in the remaining part of the paper.

Introduce a new subclass of polynomials as follows:

$$
\mathcal{P}^{t}=\mathcal{P}^{t}(\bar{q}, \bar{r})=\bigcup_{2^{t} \leqslant H<2^{t+1}} \tilde{\mathcal{P}}(H, \bar{q}, \bar{r}) .
$$


In order to proceed we need one more definition.

A polynomial $P \in \tilde{\mathcal{P}}(H, \bar{q}, \bar{r})$ is called $\left(i_{1}, i_{2}\right)$-linear, where $i_{1}=0,1$ and $i_{2}=0,1$, according to the ordering between $q_{1}+k_{2} T^{-1}$ and $v_{1}+1, r_{1}+l_{2} T^{-1}$ and $v_{2}+1$. For example, $(0,0)$-linearity means that the following system holds:

$$
\begin{aligned}
& q_{1}+k_{2} T^{-1}<v_{1}+1, \\
& r_{1}+l_{2} T^{-1}<v_{2}+1 .
\end{aligned}
$$

$(0,1)$-linearity means $(<, \geqslant)$ inequalities in the system above, $(1,1)$ linearity means $(\geqslant, \geqslant)$, and so on. The most important case are the $(1,1)$ and $(0,0)-$ linearities. Denote

$$
d_{1}=q_{1}+r_{1}, \quad d_{2}=\left(k_{2}+l_{2}\right) T^{-1} .
$$

We will consider polynomials $P \in \mathcal{P}^{t}$ such that $H \asymp Q$. The main differences between 0 - and 1-linearity will be finding proper estimates of the differences $\left|x-\alpha_{1}\right|$ and $\left|y-\beta_{1}\right|$ when applying Lemma 2. We use the first estimate in (13) for 0 -linearity and the second estimate in (13) for 1-linearity.

Proposition 2. Let $M_{n, 2}(\bar{c}, \bar{v}, Q)$ denote the set of $(x, y) \in I_{1} \times I_{2}$ such that the system of inequalities

$$
\left\{\begin{array}{l}
|P(x)| \ll Q^{-v_{1}} \\
|P(y)| \ll Q^{-v_{2}}
\end{array}\right.
$$

holds for (1,1)-linearity. Then

$$
\mu M_{n, 2}(\bar{c}, \bar{v}, Q)<\frac{1}{32}\left|I_{1}\right|\left|I_{2}\right|
$$

Proof.

$(1,1)$-linearity implies $d_{1}+d_{2} \geq n+1$. By Lemmas 2 and 3 ,

$$
\left\{\begin{array}{l}
\left|x-\alpha_{1}\right| \ll Q^{-\frac{v_{1}+1}{2}+\frac{q_{1}}{2}+(n-1) \varepsilon_{1}}, \\
\left|y-\beta_{1}\right| \ll Q^{-\frac{v_{2}+1}{2}+\frac{r_{1}}{2}+(n-1) \varepsilon_{1}} .
\end{array}\right.
$$

Suppose $\rho_{1}=\frac{v_{1}-q_{2}+1}{2}$. Let us divide the interval $I_{1}$ into equal subintervals $I_{i}$, where $\left|I_{i}\right|=Q^{-\rho_{1}+\varepsilon}$. Similarly, suppose $\rho_{2}=\frac{v_{2}-r_{2}+1}{2}$ and divide $I_{2}$ into equal subintervals $I_{j}$, where $\left|I_{j}\right|=Q^{-\rho_{2}+\varepsilon}$.

Then the number of rectangles $I_{i} \times I_{j}$ does not exceed

$$
c(n) Q^{\frac{1}{2}\left(v_{1}+v_{2}+2\right)-q_{2}-r_{2}-2 \varepsilon}\left|I_{1}\right|\left|I_{2}\right|=c(n) Q^{\frac{1}{2}(n+1)-q_{2}-r_{2}-2 \varepsilon}\left|I_{1}\right|\left|I_{2}\right| .
$$


Choose rectangles $I_{i} \times I_{j}$ that contain not more than one solution $P$ of system (39). From (41) and (42) it follows that the measure of the solution set of (39) does not exceed

$$
c(n) Q^{-2 \varepsilon+2(n-1) \varepsilon_{1}}\left|I_{1}\right|\left|I_{2}\right|<\frac{1}{64}\left|I_{1}\right|\left|I_{2}\right| .
$$

Let us show that the case where (39) holds for at least two polynomials leads to a contradiction. Using a Taylor expansion on $I_{i}$ and $I_{j}$, we obtain

$$
\begin{aligned}
& P_{1}(x)=P^{\prime}\left(\alpha_{1}\right)\left(x-\alpha_{1}\right)+\frac{1}{2} P^{\prime \prime}\left(\alpha_{1}\right)\left(x-\alpha_{1}\right)^{2}+\sum_{j=3}^{n}(j !)^{-1} P^{(j)}\left(\alpha_{1}\right)\left(x-\alpha_{1}\right)^{j}, \\
& P_{1}(y)=P^{\prime}\left(\beta_{1}\right)\left(y-\beta_{1}\right)+\frac{1}{2} P^{\prime \prime}\left(\beta_{1}\right)\left(y-\beta_{1}\right)^{2}+\sum_{j=3}^{n}(j !)^{-1} P^{(j)}\left(\beta_{1}\right)\left(y-\beta_{1}\right)^{j} .
\end{aligned}
$$

Similarly we obtain an expansion for $P_{2}$. The above estimates of $\left|x-\alpha_{1}\right|$, $\left|y-\beta_{1}\right|$, and the estimates for the derivatives that follow from Lemma 3 lead to the following inequalities:

$$
\left\{\begin{array}{l}
\left|P_{1}(x)\right| \ll Q^{-v_{1}+(n-1) \varepsilon_{1}+2 \varepsilon}, \\
\left|P_{1}(y)\right| \ll Q^{-v_{2}+(n-1) \varepsilon_{1}+2 \varepsilon} \\
\left|P_{2}(x)\right| \ll Q^{-v_{1}+(n-1) \varepsilon_{1}+2 \varepsilon} \\
\left|P_{2}(y)\right| \ll Q^{-v_{2}+(n-1) \varepsilon_{1}+2 \varepsilon}
\end{array}\right.
$$

Since $P_{1}$ and $P_{2}$ are irreducible they have no common roots. Thus, we can apply Lemma 4 to obtain

$\tau_{1}+1=v_{1}-(n-1) \varepsilon_{1}-2 \varepsilon, 2\left(\tau_{1}+1-\eta_{1}\right)=v_{1}+1+q_{2}+2(n-1) \varepsilon_{1}-4 \varepsilon$,

$\tau_{2}+1=v_{2}-(n-1) \varepsilon_{1}-2 \varepsilon, 2\left(\tau_{2}+1-\eta_{2}\right)=v_{2}+1+r_{2}+2(n-1) \varepsilon_{1}-4 \varepsilon$,

and in the left side of the inequality in Lemma 4 we get

$$
2 v_{1}+2 v_{2}+4-12 \varepsilon-6(n-1) \varepsilon_{1}=2 n+2-12 \varepsilon-6(n-1) \varepsilon_{1} .
$$

The right-hand side of this inequality then becomes $2 n+\delta$. Given $\varepsilon, \varepsilon_{1}$, we obtain a contradiction to Lemma 4 when $\delta<0.5$.

Now let consider the case of $(0,0)$ - linearity. Suppose that $n+0.1<$ $d_{1}+d_{2}<n+1$, namely

$$
\left\{\begin{array}{l}
q_{1}+k_{2} T^{-1} \leqslant v_{1}+1 \\
r_{1}+l_{2} T^{-1} \leqslant v_{2}+1 \\
d_{1}+d_{2}>n+0.1
\end{array}\right.
$$


Proposition 3. Let $M_{n, 3}(\bar{c}, \bar{v}, Q)$ denote the set of $(x, y) \in I_{1} \times I_{2}$ such that (39) holds together with (45). Then

$$
\mu M_{n, 3}(\bar{c}, \bar{v}, Q)<\frac{1}{32}\left|I_{1}\right|\left|I_{2}\right|
$$

Proposition 3 can be proved in a similar manner. When (45) holds the first estimate is sharper then the second one in (13).

Again divide the rectangle $I_{1} \times I_{2}$ into equal rectangles $I_{i} \times I_{j}$, where $\left|I_{i}\right|=Q^{-\rho_{3}+\varepsilon},\left|I_{j}\right|=Q^{-\rho_{4}+\varepsilon}$ and $\rho_{3}=k_{2} T^{-1}, \rho_{4}=l_{2} T^{-1}$. Then the number of rectangles $I_{i} \times I_{j}$ does not exceed

$$
c(n) Q^{\left(k_{2}+l_{2}\right) T^{-1}-2 \varepsilon}\left|I_{1}\right|\left|I_{2}\right| .
$$

Again choose rectangles $I_{i} \times I_{j}$ such that there are no solutions or there is at most one solution $P$ of the system (39) with an extra condition (45). By Lemma 2, we have for fixed a polynomial $P(t)$

$$
\left\{\begin{array}{l}
\left|x-\alpha_{1}\right| \ll Q^{-v_{1}-1+q_{1}+(n-1) \varepsilon_{1}}, \\
\left|y-\beta_{1}\right| \ll Q^{-v_{2}-1+r_{1}+(n-1) \varepsilon_{1}} .
\end{array}\right.
$$

Their product gives us an upper estimate for the measure of $\{(x, y): x \in$ $\left.S\left(\alpha_{1}\right), y \in S\left(\beta_{1}\right)\right\}$. Multiplying it by (47), we get the following upper estimate for the measure of the solution set:

$$
c(n) Q^{-v_{1}-v_{2}-2+\left(k_{2}+l_{2}\right) T^{-1}+q_{1}+r_{1}-2 \varepsilon+2(n-1) \varepsilon}\left|I_{1}\right|\left|I_{2}\right| \ll Q^{-\varepsilon}\left|I_{1}\right|\left|I_{2}\right|<\frac{1}{32}\left|I_{1}\right|\left|I_{2}\right| .
$$

Assume that there are at least two solutions in the rectangle $I_{1} \times I_{2}$. Again using a Taylor expansion of $P$ and estimating its summands from above we obtain

$$
\left\{\begin{array}{l}
\left|P_{1}(x)\right| \ll Q^{1-q_{1}-k_{2} T^{-1}+(n-1) \varepsilon_{1}-\varepsilon} \\
\left|P_{1}(y)\right| \ll Q^{1-r_{1}-l_{2} T^{-1}+(n-1) \varepsilon_{1}-\varepsilon} \\
\left|P_{2}(x)\right| \ll Q^{1-q_{1}-k_{2} T^{-1}+(n-1) \varepsilon_{1}-\varepsilon} \\
\left|P_{2}(y)\right| \ll Q^{1-r_{1}-l_{2} T^{-1}+(n-1) \varepsilon_{1}-\varepsilon}
\end{array}\right.
$$

By Lemma 4 for

$$
\begin{aligned}
& \tau_{1}+1=q_{1}+k_{2} T^{-1}-(n-1) \varepsilon_{1}-\varepsilon, \quad 2\left(\tau_{1}+1-\eta_{1}\right)=2 q_{1}-2(n-1) \varepsilon_{1}-2 \varepsilon, \\
& \tau_{2}+1=r_{1}+l_{2} T^{-1}-(n-1) \varepsilon_{1}-\varepsilon, \quad 2\left(\tau_{2}+1-\eta_{2}\right)=2 r_{1}-2(n-1) \varepsilon_{1}-2 \varepsilon,
\end{aligned}
$$

we get the following left-hand side for the inequality in Lemma 4

$$
3 q_{1}+k_{2} T^{-1}+3 r_{1}+l_{2} T^{-1}-6(n-1) \varepsilon_{1}-6 \varepsilon .
$$


But $k_{2} T^{-1} \leqslant q_{1}, l_{2} T^{-1} \leqslant r_{1}$, and (45) implies that the expression in (49) is at least

$$
\begin{gathered}
2\left(d_{1}+d_{2}\right)-6 \varepsilon-6(n-1) \varepsilon_{1} \geqslant 2\left(v_{1}+v_{2}\right)+3.6-6 \varepsilon-6(n-1) \varepsilon_{1}= \\
=2 n+0.2-6 \varepsilon-6(n-1) \varepsilon_{1} .
\end{gathered}
$$

Given $\varepsilon, \varepsilon_{1}$, we obtain a contradiction to Lemma 4 when $\delta<0.1$.

Now let us consider the case of $(0,0)$-linearity for

$$
n-0.3<d_{1}+d_{2} \leq n+0.1
$$

Proposition 4. Let $M_{n, 4}(\bar{c}, \bar{v}, Q)$ denote the set of $(x, y) \in I_{1} \times I_{2}$ such that (38), (39) hold together with (50). Then

$$
\mu M_{n, 4}(\bar{c}, \bar{v}, Q)<\frac{1}{32}\left|I_{1}\right|\left|I_{2}\right| .
$$

Proof.

Let us divide the rectangle $I_{1} \times I_{2}$ into equal rectangles $I_{i} \times I_{j}$, where $\left|I_{i}\right|=Q^{-k_{2} T^{-1}-\gamma_{1}},\left|I_{j}\right|=Q^{-l_{2} T^{-1}-\gamma_{1}}$ for some $\gamma_{1}>0$ that will be specified below. Let us choose those rectangles where the system (39) has at least $c(n) Q^{\theta_{1}}$ solutions in polynomials $P(t)$ for some $\theta_{1} \geq 0$. Estimate the measure of $A_{1}=\left\{(x, y):(x, y) \in I_{i} \times J_{j}\right\}$, which satisfies (39).

$$
\begin{gathered}
\mu A_{1} \ll Q^{-v_{1}-1+q_{1}-v_{2}-1+r_{1}+k_{2} T^{-1}+l_{2} T^{-1}+2 \gamma_{1}+\theta_{1}}\left|I_{1}\right| \times\left|I_{2}\right| \ll \\
\ll Q^{\theta_{1}-n-1+d_{1}+d_{2}+2 \gamma_{1}}\left|I_{1}\right|\left|I_{2}\right| .
\end{gathered}
$$

When

$$
\theta_{1}<n+1-d_{1}-d_{2}-2 \gamma_{1}
$$

the statement of Proposition 4 can be easily verified.

Consider now the opposite inequality

$$
\theta_{1} \geq u_{1}=n+1-d_{1}-d_{2}-2 \gamma_{1} \text {. }
$$

By $(50), \theta_{1}>0$ for $\gamma_{1} \leq 0.4$.

Similarly to (48), estimate $P_{l}(t), l=1,2$, in $I_{i} \times J_{j}$. We obtain

$$
\begin{gathered}
\left|P_{l}(x)\right| \ll Q^{1-q_{1}-k_{2} T^{-1}-\gamma_{1}+(n-1) \varepsilon_{1}}, \\
\left|P_{l}(y)\right| \ll Q^{1-r_{1}-l_{2} T^{-1}-\gamma_{1}+(n-1) \varepsilon_{1}} .
\end{gathered}
$$


Apply Lemma 4 to $P_{1}(t)$ and $P_{2}(t)$ with following parameters

$$
\begin{gathered}
\tau_{1}+1=q_{1}+k_{2} T^{-1}+\gamma_{1}-(n-1) \varepsilon_{1}, \\
2\left(\tau_{1}+1-\eta_{1}\right)=2 q_{1}-2(n-1) \varepsilon_{1}, \\
\tau_{2}+1=r_{1}+l_{2} T^{-1}+\gamma_{1}-(n-1) \varepsilon_{1}, \\
2\left(\tau_{2}+1-\eta_{2}\right)=2 r_{1}-2(n-1) \varepsilon_{1} .
\end{gathered}
$$

By Lemma 4 and (50), the inequality

$$
2\left(d_{1}+d_{2}\right)+0.8-6(n-1) \varepsilon_{1}<2 n+\delta
$$

leads to a contradiction.

Consider now the next case when

$$
n-0.55<d_{1}+d_{2} \leq n-0.3 .
$$

Proposition 5. Let $M_{n, 5}(\bar{c}, \bar{v}, Q)$ denote the set of $(x, y) \in I_{1} \times I_{2}$ such that (38), (39) hold together with (56). Then

$$
\mu M_{n, 5}(\bar{c}, \bar{v}, Q)<\frac{1}{32}\left|I_{1}\right|\left|I_{2}\right|
$$

Proof.

The proof of Proposition 5 is similar to the proof of Proposition 4. Let us divide the rectangle $I_{1} \times I_{2}$ into equal rectangles $I_{i} \times I_{j}$, where $\left|I_{i}\right|=$ $Q^{-k_{2} T^{-1}-\gamma_{2}},\left|I_{j}\right|=Q^{-l_{2} T^{-1}-\gamma_{2}}$ for some $\gamma_{2}>0$. Similarly, we introduce a constant $\theta_{2} \geq 0$ and a set $A_{2}$. When $\theta_{2}<n+1-d_{1}-d_{2}-2 \gamma_{2}$ holds, then Proposition 4 can be easily proved. So consider

$$
\theta_{2} \geq u_{2}=n+1-d_{1}-d_{2}-2 \gamma_{2}
$$

By (56), we can choose $\gamma_{2}=0.6$ in (58). Similarly to (53), estimate $P_{l}(t)$, $l=1,2$ in newly constructed rectangles $I_{i} \times J_{j}$. Applying Lemma 4 , we obtain an inequality similar to (55)

$$
2\left(d_{1}+d_{2}\right)+1.2-6(n-1) \varepsilon_{1}<2 n+\delta .
$$

Since (56) and $\delta<0.05$, the inequality leads to a contradiction.

Let

$$
2<d_{1}+d_{2} \leq n-0.55 .
$$

Proposition 6. Let $M_{n, 6}(\bar{c}, \bar{v}, Q)$ denote the set of $(x, y) \in I_{1} \times I_{2}$ such that (38), (39) hold together with (59). Then

$$
\mu M_{n, 5}(\bar{c}, \bar{v}, Q)<\frac{1}{32}\left|I_{1}\right|\left|I_{2}\right|
$$


Proof.

The start of the proof is similar to the proofs of Propositions 4 and 5 . We divide the rectangle $I_{1} \times I_{2}$ into equal rectangles $I_{i} \times I_{j}$, where $\left|I_{i}\right|=Q^{-k_{2} T^{-1}}$, $\left|I_{j}\right|=Q^{-l_{2} T^{-1}}$. Similarly, we introduce the constant $\theta_{3} \geq 0$ and the set $A_{3}$. When $\theta_{3}<n+1-d_{1}-d_{2}$ holds the proof of Proposition 6 is obvious. Consider now

$$
\theta_{3} \geq u_{3}=n+1-d_{1}-d_{2} \geq 1.45 .
$$

We can rewrite $u_{3}$ as

$$
u_{3}=\left[u_{3}\right]+\left\{u_{3}\right\}, \quad\left[u_{3}\right] \geq 1 .
$$

Expanding $P_{l}(t)$ and $P_{l}^{\prime}(t)$ on intervals $I_{j}$ and $J_{i}$ into a Taylor series and estimating its terms above, we obtain

$$
\left\{\begin{array}{l}
|P(x)| \ll Q^{1-q_{1}-k_{2} T^{-1}} \\
\left|P^{\prime}(x)\right| \ll Q^{1-q_{1}}, \\
|P(y)| \ll Q^{1-r_{1}-l_{2} T^{-1}} \\
\left|P^{\prime}(y)\right| \ll Q^{1-r_{1}}
\end{array}\right.
$$

Since there are at most $c(n) Q^{\left[u_{3}\right]+\left\{u_{3}\right\}}$ polynomials $P(t)$ that belong to $I_{j} \times J_{i}$, then, by Dirichlet's principle, there are at least $K=c(n) Q^{\left\{u_{1}\right\}}$ polynomials with equal coefficients of $t^{n}, t^{n-1}, \ldots, t^{n-\left[u_{3}\right]+1}$.

Now we construct further polynomials with degree at most $n-\left[u_{3}\right]$

$$
R_{j-1}(t)=P_{j}(t)-P_{1}(t) \quad j=2, \ldots, K .
$$

By (62) for $R_{i}(f), i=1, \ldots, K-1$, we have

$$
\left\{\begin{array}{l}
\left|R_{i}(x)\right| \ll Q^{1-q_{1}-k_{2} T^{-1}+(n-1) \varepsilon_{1}}, \\
\left|R^{\prime}(x)\right| \ll Q^{1-q_{1}}, \\
\left|R_{i}(y)\right| \ll Q^{1-r_{1}-l_{2} T^{-1}+(n-1) \varepsilon_{1}}, \\
\left|R^{\prime}(y)\right| \ll Q^{1-r_{1}} \\
\operatorname{deg} R_{i} \leq n-\left[u_{3}\right]=d_{1}+d_{2}+\left\{u_{3}\right\}-1 .
\end{array}\right.
$$

We apply Lemma 4 to the two polynomials $R_{s_{1}}(t)$ and $R_{s_{2}}(t)$. This results in a contradiction when $\left\{u_{3}\right\} \leq 0.7$.

Thus assume that $\left\{u_{3}\right\}>0.7$. Again we divide the rectangle $I_{1} \times I_{2}$ into equal rectangles $I_{i} \times I_{j}$, where $\left|I_{i}\right|=Q^{-k_{2} T^{-1}-\gamma_{3}},\left|I_{j}\right|=Q^{-l_{2} T^{-1}-\gamma_{3}}$ for some $\gamma_{3}>0$ such that $2 \gamma_{3} \leq\left\{u_{3}\right\}$. If the number of polynomials in these 
rectangles is $c(n) Q^{\theta_{3}}$ and $\theta_{3}<u_{3}=n+1-d_{1}-d_{2}-2 \gamma_{3}$ then Proposition 6 can be easily proved. When

$$
\theta_{3} \geq u_{3}=n+1-d_{1}-d_{2}-2 \gamma_{3}=\left[u_{3}\right]+\left\{u_{3}\right\} n-2 \gamma_{3}
$$

one can obtain (63) with an approximation of $\left|R_{i}(x)\right|$ and $\left|R_{i}(y)\right|$ of the type $1-q_{1}-k_{2} T^{-1}-\gamma_{3}+(n-1) \varepsilon_{1}$ and $1-r_{1}-l_{2} T^{-1}-\gamma_{3}+(n-1) \varepsilon_{1}$ respectively. Applying Lemma 4 to the pair of coprime polynomials, we get

$$
2\left(d_{1}+d_{2}\right)-6(n-1) \varepsilon_{1}+2 \gamma_{3}<2\left(d_{1}+d_{2}\right)-2+2\left\{u_{4}\right\}+\delta
$$

that leads to a contradiction for $\gamma_{3}=\frac{\left\{u_{4}\right\}}{2}$ and $\delta=0.1$.

Let us show how the theorem can be proved for the cases of $(1,0)$ and $(0,1)$-linearity. Since both proofs are absolutely similar we will demonstrate the method for $(1,0)$-linearity only.

Proposition 7. Let $M_{n, 7}(\bar{c}, \bar{v}, Q)$ denote the set of $(x, y) \in I_{1} \times I_{2}$ such that (39) hold together with

$$
\left\{\begin{array}{l}
q_{1}+k_{2} T^{-1}>v_{1}+1 \\
r_{1}+l_{2} T^{-1} \leq v_{2}+1
\end{array}\right.
$$

Then

$$
\mu M_{n, 7}(\bar{c}, \bar{v}, Q)<\frac{1}{32}\left|I_{1}\right|\left|I_{2}\right|
$$

Proof.

Again divide the rectangle $I_{1} \times I_{2}$ into rectangles $I_{i} \times I_{j}$, where $\left|I_{i}\right|=$ $Q^{-\frac{v_{1}-q_{2}+1}{2}+\varepsilon},\left|I_{j}\right|=Q^{-l_{2} T^{-1}+\varepsilon}$. We replace the second inequality in (64) by

$$
v_{2}+0.5<r_{1}+l_{2} T^{-1} \leq v_{2}+1 \text {. }
$$

Consider the rectangles $I_{i} \times I_{j}$ which contain no more than one polynomial $P(t)$. Fix such a polynomial $P(t)$. Then the solution of (39) belongs to the rectangle

$$
\left\{\begin{array}{l}
|x-\alpha| \ll Q^{-\frac{v_{1}+1-q_{2}}{2}}, \\
|y-\beta| \ll Q^{-v_{2}-1+r_{1}} .
\end{array}\right.
$$

Multiplying the estimates (66), we sum them over all rectangles $I_{i} \times I_{j}$. Thus we get the estimate of the kind $c(n) Q^{-\varepsilon}\left|I_{1}\right|\left|I_{2}\right|$ that proves Proposition 7. If there are at least two polynomials such that belong to $I_{i} \times I_{j}$, then we expand them into Taylor series. We get

$$
\left|P_{i}(x)\right| \ll Q^{-v_{1}+(n-1) \varepsilon_{1}+2 \varepsilon},
$$




$$
\left|P_{i}(y)\right| \ll Q^{1-r_{1}-l_{2} T^{-1}}
$$

Apply Lemma 4 with

$$
\begin{gathered}
\tau_{1}=v_{1}+1-2 \varepsilon-(n-1) \varepsilon_{1}, \\
2\left(\tau_{1}+1-\eta_{1}\right)=v_{1}+1+q_{2}-2 \varepsilon-2(n-1) \varepsilon_{1}, \\
\tau_{2}+1=r_{1}+l_{2} T^{-1}-\varepsilon, \\
2\left(\tau_{2}+1-\eta_{2}\right)=2 r_{1} .
\end{gathered}
$$

Then,

$$
2 v_{1}+2+l_{2} T^{-1}+3 r_{1}+q_{2}-3(n-1) \varepsilon_{1}-4 \varepsilon<2 n+\delta .
$$

However, by (65), we have $l_{2} T^{-1}+3 r_{1}>2 v_{2}+1$, and the left side in (67) is larger than $2 n+1-5 \varepsilon$. Thus, for $\delta<0.5$ we arrive at a contradiction.

The final part of the proof is similar to the proof of the $(0,0)$-linearity. We omit the above estimate in (65) until we can use Dirichlet's principle, which results in polynomials of lower degree.

The case $r_{1}<\frac{1}{2}$ and $r_{1}<\frac{1}{2}$ is considered in Proposition 1. It remains to consider polynomials such that

$$
1 \leq d_{1}+d_{2} \leq 2
$$

holds. Here as in Proposition 1 we can pass to first degree polynomials which lead to a contradiction with (3) or to the second degree polynomials. For this case Theorem 1 was proved in Proposition 1.

Combining the results of all Propositions, we finally get

$$
\mu M_{n}(\bar{c}, \bar{v}, Q) \leqslant \sum_{j=1}^{7} \mu M_{n, j}(\bar{c}, \bar{v}, Q) \leqslant \frac{1}{4}\left|I_{1}\right|\left|I_{2}\right|,
$$

concluding the proof of Theorem 2 .

\section{References}

[1] J. Cassels, An introduction to Diophantine approximation. Cambridge Tracts in Mathematics and Mathematical Physics, No. 45. Cambridge University Press, New York, 1957.

[2] W. Schmidt, Diophantine approximation. Lecture Notes in Mathematics, 785. Springer, Berlin, 1980.

[3] A. Karatsuba, Basic analytic number theory. Springer-Verlag, Berlin, 1993. 
[4] M. Huxley, Area, lattice points, and exponential sums. London Mathematical Society Monographs. New Series, 13. Oxford Science Publications. The Clarendon Press, Oxford University Press, New York, 1996.

[5] F. Götze, Lattice point problems and values of quadratic forms. Invent. Math. 157 (2004), no. 1, 195-226.

[6] R. Vaughan, S. Velani, Diophantine approximation on planar curves: the convergence theory. Invent. Math. 166 (2006), no. 1, 103-124.

[7] V. Beresnevich, D. Dickinson, S. Velani, Diophantine approximation on planar curves and the distribution of rational points. With an Appendix II by R. C. Vaughan. Ann. of Math. (2) 166 (2007), no. 2, 367-426.

[8] V.G. Sprindzuk, Mahler's problem in metric Number Theory // Nauka i Tehnika, Minsk 1967 [Transl. Math. Monogr. 25, Amer. Math. Soc., Providenca, R.I., 1969].

[9] V.I. Bernik, M.M. Dodson, Metric Diophantine approximation on manifolds // Cambridge Tracts in Math. Vol. 137. Cambridge Univ. Press, 1999.

[10] $V$. Bernik, The metric theorem on the simultaneous approximation of zero by values of integral polynomials // Izv. Akad. Nauk SSSR, Ser. Mat. 44, 1980, P. $24-45$.

[11] $V$. Bernik, On the exact order of approximation of zero by values of integral polynomials // Acta Arith. 53, 1989, P. $17-28$.

Institute of Mathematics

Surganova str. 11

220072, Minsk,

Belarus

E-mail: bernik@im.bas-net.by olga_kukso@tut.by
Universität Bielefeld

Fakultät für Mathematik

Postfach 100131

33501 Bielefeld

Germany

E-mail: goetze@math.uni-bielefeld.de 